\title{
La rétention des protéines dans le réticulum endoplasmique
}

La cellule eucaryote est divisée en compartiments intracellulaires délimités par des membranes. Chaque compartiment contient des constituants cellulaires, protéines et lipides, qui lui sont propres et y remplissent des fonctions spécifiques. Cette compartimentation implique donc un mécanisme de ségrégation des constituants cellulaires qui doivent être insérés dans le compartiment approprié. Les protéines, en particulier, doivent être acheminées depuis leur lieu de synthèse sur les ribosomes du cytosol jusqu'à leur destination finale dans l'un ou l'autre de ces compartiments cellulaires.

La voie vacuolaire centrale constitue une artère particulièrement encombrée de la circulation des protéines dans la cellule. Les protéines empruntant cette voie sont d'abord insérées dans la membrane ou en solution dans la lumière du réticulum endoplasmique. De là, les protéines sont ensuite transportées vers l'appareil de Golgi, les lysosomes, les granules de sécrétion ou la surface cellulaire. On admet généralement que l'insertion dans une des grandes voies du transport intracellulaire, ou sa rétention dans un compartiment cellulaire, dépendent de la présence d'un signal dans la structure de cette protéine. Ce signal est reconnu par une machinerie chargée de trier les protéines et de les acheminer jusqu'à leur destination finale. Une protéine dépourvue de signal serait acheminée librement jusqu'à la surface cellulaire qui semble ainsi constituer la voie par défaut du transport intracellulaire.

L'appareil de Golgi semble constituer une plaque tournante dans la voie vacuolaire centrale et serait le lieu où sont prises la plupart des décisions concernant le routage des $\mathrm{m} / \mathrm{s} n^{\circ} 11$ vol. 9, novembre 93 protéines empruntant cette voie. Ainsi, les hydrolases acides quittent les saccules trans de l'appareil de Golgi dans des vésicules qui iront fusionner avec les lysosomes [1]. Les enzymes de la glycosylation sont insérées, soit dans les différentes saccules de l'appareil de Golgi, soit, pour les enzymes impliquées dans les premières étapes de la glycosylation, dans le réticulum endoplasmique. C'est dans cet organite que se retrouvent aussi les protéines aidant au repliement de la chaîne peptidique en croissance. Cette classe de protéines résidentes du réticulum endoplasmique est particulièrement intéressante et on commence à comprendre le mécanisme qui les retient dans cet organite. Parmi ces protéines, on retrouve la $\mathrm{BiP}$ (immunoglobulin binding protein, Grp78), la Grp94 (glucose-related protein $94 \mathrm{kDa}$ ) et la PDI (protein disulfide isomerase).

\section{Le signal}

L'examen des séquences de plusieurs protéines retenues en solution dans le réticulum a permis de constater qu'elles possédaient toutes à leur extrémité C-terminale le tétrapeptide KDEL (Lys-Asp-Glu-Leu) ([2], $\mathrm{m} / \mathrm{s} n^{\circ} 7$, vol. 3, p. 423; $\mathrm{m} / \mathrm{s}$ $n^{\circ} 7$, vol. $\left.6, p .702\right)$. Cette observation a été le point de départ d'une série d'expériences faisant appel aux techniques de biologie moléculaire et permettant de déterminer la localisation de protéines mutantes ou chimères après transfection dans un système hétérologue. Cette localisation est établie par immunofluorescence, ou par marquage métabolique suivi d'immunoprécipitation et d'une analyse électrophorétique. En immunofluorescence, on observe un marquage fibrillé qui est typique du réticulum endoplasmique; le marquage métabolique, suivi d'immuno- précipitation, permet de distinguer une protéine du réticulum d'une protéine transportée plus en aval de la voie vacuolaire centrale, en vérifiant qu'elle n'a pas subi l'action des mannosidases des saccules médians de l'appareil de Golgi. La délétion de la courte séquence KDEL provoque la sécrétion des protéines mutées. De plus, l'ajout du tétrapeptide KDEL à l'extrémité C-terminale du lysozyme entraîne la rétention de cette protéine dont la forme non mutée est normalement sécrétée. Ces observations indiquent que le tétrapeptide KDEL agit comme signal de rétention au réticulum endoplasmique lorsqu'il est placé à l'extrémité C-terminale d'une protéine.

Dans les premières expériences du groupe de Pelham, le signal de rétention dans la levure Saccharomyces cerevisiae (HDEL*) fut ajouté à l'extrémité C-terminale du lysozyme. Cependant, cette addition n'entraîna pas la rétention de la protéine chimère dans le réticulum endoplasmique de cellules de mammifères. Les auteurs en conclurent que le signal de rétention des protéines de mammifères n'était pas interchangeable avec celui des protéines de la levure. Quelques données récentes sont venues contredire ce résultat. En effet, Robbi et Beaufay [3] ont montré que différentes carboxylestérases de mammifères avec pour extrémité C-terminale HIEL*, HTEL* et HVEL* sont retenues dans le réticulum endoplasmique lorsqu'elles sont transfectées dans des cellules COS.

\footnotetext{
* Code à une lettre des acides aminés: A : Ala $C: C y s ; D: A s p ; E: G l u ; F: P h e ; G: G l y ; H$ His; I :lle; $K:$ Lys; L : Leu; $M:$ Met; $N: A s n$; $P:$ Pro; $Q: G l n ; R: A r g ; S: S e r ; T: T h r ; V:$ Val; W: Trp; Y: Tyr.
} 
La séquence HVEL de l'estérase pI 6,4 peut être mutée en HDEL sans affecter pour autant la rétention de l'enzyme. En revanche, le mutant HVE $R^{*}$ n'est pas retenu : $50 \%$ des protéines sont sécrétées 3 heures après leur synthèse. Cette sécrétion est lente mais significative: un mutant PDI dont l'extrémité KDEL* a été changée en QEDL* est sécrété à la même vitesse [4]. D'ailleurs, il est intéressant de noter ici que l'addition de la séquence SEKDEL* à deux protéines normalement sécrétées, l'hormone de croissance et la sous-unité $\alpha$ de la gonadotropine chorionique, ne conduit pas à la rétention des protéines mutantes dans le réticulum endoplasmique mais entraîne plutôt une diminution dramatique de la vitesse de sécrétion [4]. Bien que la cause majeure de la rétention dans le réticulum soit la présence du tétrapeptide Cterminal KDEL, la conformation protéique autour de ce signal semble aussi affecter l'efficacité du processus [5].

De leur côté, Andres et al. [6], cherchant à définir un consensus pour le signal de rétention, ont établi une série de lignées AtT-20 exprimant des constructions de diverses séquences C-terminales fusionnées à un gène rapporteur, le préproneuropeptide Y. L'addition des tétrapeptides KDEL, QDEL, KEDL et KDEI inhibe la maturation et la sécrétion des neuropeptides Y mutants. L'observation par immunofluorescence de ces mutants marqués suggère une localisation au niveau du réticulum endoplasmique. En revanche, la distribution intracellulaire, la maturation et la sécrétion de la chimère prépro-neuropeptide Y-KDEV* sont similaires à celles du précurseur non muté. A la lumière de ces résultats, les auteurs proposent qu'un signal de rétention dans le réticulum endoplasmique doit contenir un acide aminé chargé négativement (acide aspartique ou glutamique) à l'avant-dernière position du tétrapeptide et une large chaîne latérale aliphatique (leucine ou isoleucine) à la dernière position.

\section{Le mécanisme}

D'après l'analyse des schémas de phosphorylation, Pelham avait montré qu'une chimère cathepsine-DSEKDEL passait par le cis-Golgi avant de retourner au réticulum endoplasmique [7]. En effet, la protéine mutante peut être marquée au phosphate radioactif et ce marquage disparaît après traitement à l'endoglycosidase $H$, suggérant une action de la N-acétylglucosaminyl-lphosphotransférase, un marqueur du cis-Golgi. Pelham proposait alors que la rétention dans le réticulum est un processus dynamique qui implique l'action d'un récepteur reconnaissant spécifiquement les protéines munies du signal KDEL à leur sortie du réticulum endoplasmique. L'interaction ligand-récepteur entraînerait la formation de vésicules de transport vers le réticulum endoplasmique. Ce mécanisme serait semblable à celui décrit pour l'endocytose [8].

\section{Le récepteur}

L'analyse de souches mutantes de levure incapables de retenir les protéines du réticulum endoplasmique (BiP, par exemple, est sécrété) a

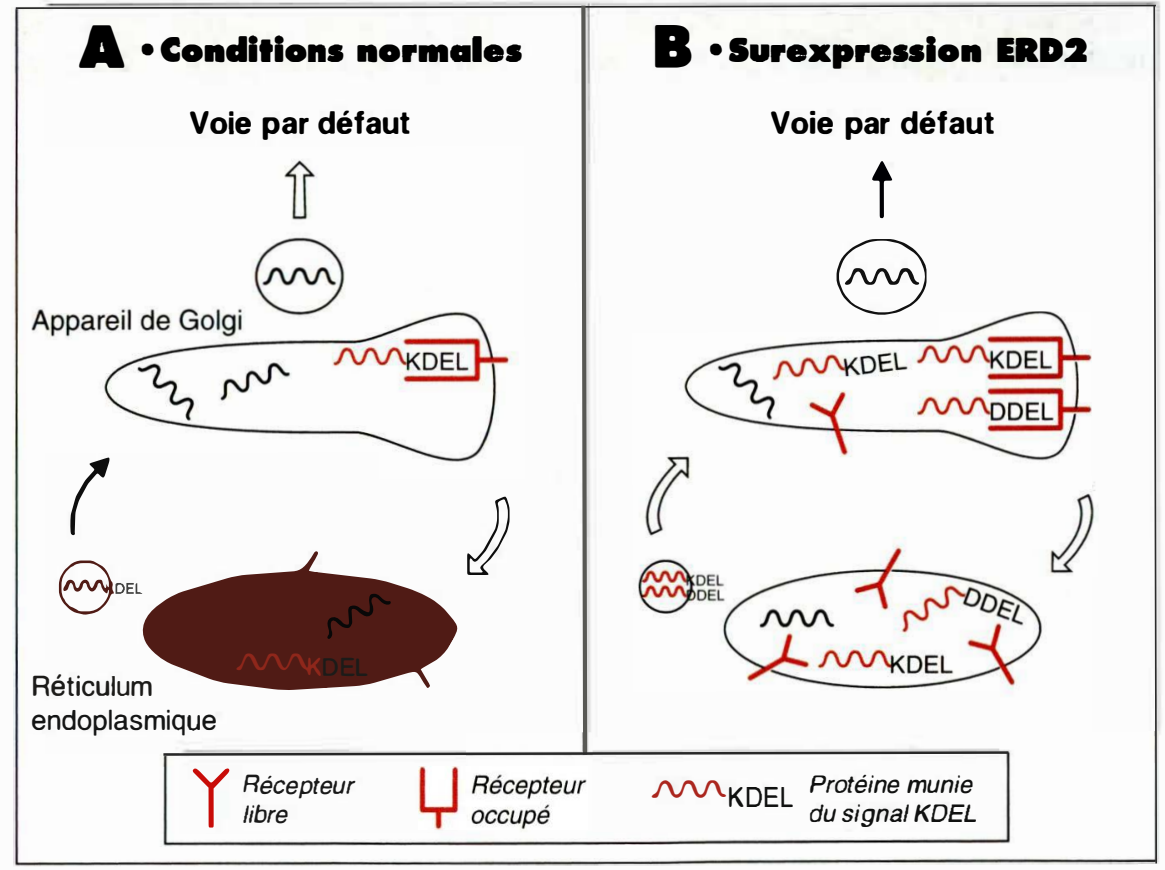

Figure 1. La rétention des protéines dans le réticulum endoplasmique. A. Conditions normales : les protéines munies du signal KDEL et le récepteur ERD2 voyagent ensemble du réticulum endoplasmique au cis-Golgi. Selon le modèle actuel, la liaison du ligand induit un changement de conformation du récepteur. Cet effet entraîne la formation de vésicules destinées à la voie rétrograde, c'est-à-dire le retour des protéines KDEL dans la lumière du réticulum endoplasmique et le recyclage du récepteur KDEL. $\mathbf{B}$. Surexpression du récepteur: la surexpression du récepteur ERD2 augmente la capacité de rétention du système et permet la capture de protéines normalement faiblement retenues comme la chimère lysozyme D DEL. De plus, la surabondance du récepteur dans les saccules golgiens inhibe localement l'association des coatomers aux membranes, ce qui favorise le trafic vésiculaire vers la voie rétrograde. 
permis l'identification, par complémentation, du gène codant pour le récepteur KDEL [9]. Le gène erd2 (pour endoplasmic reticulum retention defective) code pour une protéine relativement hydrophobe de $26 \mathrm{kDa}$, non glycosylée et essentielle à la croissance. La surexpression d'une protéine HDEL sature le système de rétention et entraîne par conséquent la sécrétion partielle des protéines normalement retenues dans le réticulum. La surexpression de ERD2 empêche ces fuites de se produire, vraisemblablement en augmentant la capacité du système de rétention (figure 1). Par la technique de PCR, Lewis et Pelham ont identifié l'homologue humain du récepteur HDEL [10]. La protéine de mammifère présente $50 \%$ d'identité avec sa cousine ERD2. Les études fonctionnelles sur ce récepteur hypothétique n'ont pas tardé à suivre [11]. D'abord, comme il avait été observé chez la levure, la surexpression du récepteur permet la rétention de protéines normalement faiblement retenues, par exemple la chimère lysozyme-DDEL. Ensuite, le récepteur est localisé au niveau de l'appareil de Golgi et la surexpression de ligands KDEL cause une redistribution du récepteur vers le réticulum endoplasmique. Enfin, l'introduction de mutations précises dans la séquence du récepteur change sa spécificité. Il semble donc probable qu'il $y$ ait interaction directe entre les protéines présentant la séquence KDEL et le récepteur chargé de les rediriger vers le réticulum endoplasmique. On peut imaginer que le récepteur adopte deux conformations distinctes selon qu'il est ou non lié à un ligand. Le récepteur libre est transporté du réticulum vers l'appareil de Golgi tandis que la forme liée est reconnue par le système de transport en sens inverse (transport rétrograde). Des expériences récentes sont venues raffiner ce modèle. L'utilisation de la bréfeldine $\mathrm{A}$, une drogue qui inhibe l'association des facteurs cytosoliques de type coatomers aux vésicules de transport et entraîne la fusion de l'appareil de Golgi au réti- culum endoplasmique, a permis de distinguer les deux voies de transport hors du Golgi. La voie antérograde vers la surface, sensible à la bréfeldine A, requiert le bourgeonnement de vésicules enrobées de protéines de type coatomer, tandis que la voie rétrograde utilise des vésicules dépourvues de coatomers et n'est donc pas sensible à la bréfeldine A. L'équipe de Klausner a montré que la surexpression de ELP-1, un homologue de ERD2, mimait l'effet de la bréfeldine A [12]. Ces auteurs ont proposé que la surabondance du récepteur ELP-1 dans les saccules de l'appareil de Golgi inhibe localement l'association des facteurs coatomers aux membranes et entraîne la formation de vésicules de transport non enrobées, destinées par conséquent à la voie rétrograde [13].

Un mot sur la rétention des protéines transmembranaires dans le réticulum endoplasmique

Les six derniers acides aminés (DEKKMP*) de la queue cytosolique de la protéine adénovirale $\mathrm{E} 3 / 19 \mathrm{~K}$ sont nécessaires à la rétention de cette protéine transmembranaire dans le réticulum endoplasmique [14], où elle se fixe aux antigènes de classe I du complexe majeur d'histocompatibilité et en empêche le transport intracellulaire. $\mathrm{Si}$ on greffe la queue cytosolique de cette protéine à un marqueur de la surface cellulaire (CD8), la protéine chimère est retenue dans le réticulum endoplasmique. L'analyse détaillée d'un grand nombre de mutants CD8-E3/19K a montré que deux lysines placées en position -3 et -4 de l'extrémité de la queue cytosolique étaient essentielles à la rétention des protéines mutantes dans le réticulum endoplasmique. Le signal est fortement spécifique puisque ni une histidine, ni une arginine ne peuvent occuper l'une ou l'autre de ces positions sans entraîner une perte de la rétention.

On a suggéré qu'une interaction entre la queue cytosolique de la protéine et les éléments du cytosque- lette serait responsable de la rétention de protéines membranaires dans le réticulum endoplasmique. Une telle association a été démontrée in vitro dans le cas de la queue cytosolique de E3/19K [15].

\section{Conclusion}

Grâce aux méthodes de dissection moléculaire et au travail de plusieurs équipes, les mécanismes de la rétention des protéines solubles dans le réticulum endoplasmique sont en grande partie élucidés: les principaux acteurs, le signal KDEL et le récepteur ERD2, sont bien connus. Il reste maintenant à trouver quel signal et quel récepteur rapportent la paire KDEL-ERD2 du cis-Golgi au réticulum endoplasmique. Il y a fort à parier que ces recherches débordent sur l'ensemble du trafic membranaire et révèlent plus avant le dynamisme et l'interdépendance des organites cellulaires. Cette approche sera probablement en mesure de nous livrer les secrets des voies de ciblage vers d'autres compartiments cellulaires. Sans doute, à l'instar de la mucolipidose de type II causée par le ciblage défectueux des enzymes lysosomiales, d'autres maladies sont dues à des aberrations dans les mécanismes de tri intracellulaire 


\section{RÉFÉRENCES}

1. Crine P, Desparois L, Lecavalier H. Un code postal pour les enzymes lysosomiales. médecine/sciences 1987 ; 3: 453-60.

2. Munro S, Pelham HRB. A C-terminal signal prevents secretion of luminal ER proteins. Cell 1987; 48: 899-907.

3. Robbi M, Beaufay $\mathrm{H}$. The $\mathrm{COOH}$ terminus of several liver carboxylesterases targets these enzymes to the lumen of the endoplasmic reticulum. I Biol Chem 1991 ; 266 : 20498-503.

4. Zagouras P, Rose JK Carboxy-terminal SEKDEL sequences retard but do not retain two secretory proteins in the endoplasmic reticulum. I Cell Biol 1989; 109 : 2633-40.

5. Haugejorden SM, Srinivasan M, Green M. Analysis of the retention signals of two resident luminal endoplasmic reticulum proteins by in vitro mutagenesis. J Biol Chem 1991 ; 266 : 6015-8.

6. Andres DA, Rhodes JD, Meisel RL, Dixon J. Characterization of the carboxylterminal sequences responsible for protein retention in the endoplasmic reticulum. $J$ Biol Chem 1991 ; 266 : 14277-82.

7. Pelham HRB. Evidence that luminal ER proteins are sorted from secretory proteins in a post-ER compartment. EMBOJ 1988 ; 7: 913-8.

8. Pearse BMF, Bretscher MS. Membrane recycling by coated vesicles. Ann Rev Biochem $1981 ; 50$ : 85-101.

9. Semenza JC, Hardwick KG, Dean N, Pelham HRB. ERD2, a yeast gene required for the receptor-mediated retrieval of luminal ER proteins from the secretory pathway. Cell 1990; 61: 1349-57.

10. Lewis MJ, Pelham HRB. A human homologue of the yeast HDEL receptor. Nature 1990 ; 348: 162-3.

11. Lewis MJ, Pelham HRB. Ligandinduced redistribution of a human KDEL receptor from Golgi complex to the endoplasmic reticulum. Cell 1992 ; 68 : 353-64. 12. Hsu VW, Shah N, Klausner RD. A brefeldin A-like phenotype is induced by overexpression of a human ERD-2-like protein, ELP-1. Cell 1992; 69: 625-35.

13. Pauloin A. Bréfeldine A, protéines $G$ et transports membranaires golgiens. médecine/sciences $1993 ; 9$ : 917-25.

14. Jackson MR, Nilsson T, Peterson PA. Identification of a consensus motif for retention of transmembrane proteins in the endoplasmic reticulum. $E M B O J 1990$; 9: 3153-62.

15. Dahlloff B, Wallin M, Kvist S. The endoplasmic reticulum retention signal of the $\mathrm{E} 3 / 19 \mathrm{~K}$ protein of adenovirus-2 is microtubule binding. J Biol Chem 1991; 266: 1804-8.

\section{TIRÉS A PART}

C. Lanctôt. 